\title{
COMPRESSIBLE ENDS OF LEAVES IN FOLIATED 3-MANIFOLDS
}

\author{
CHARALAMBOS CHARITOS
}

(Received 12 April 1996; revised 23 December 1996)

Communicated by J. A. Hillman

\begin{abstract}
In this paper we study the asymptotic behavior of cylindrical ends in compact foliated 3-manifolds and give a sufficient condition for these ends to spiral onto a toral leaf.
\end{abstract}

1991 Mathematics subject classification (Amer. Math. Soc.): primary 57R30; secondary 53C12.

\section{Introduction and preliminaries}

In this paper we shall be concerned with orientable foliations $\mathscr{F}$ of dimension two of compact three dimensional orientable manifolds. If the boundary of a manifold $M$ is not empty, we always assume the components of $\partial M$ are leaves of the foliation, and all foliations and maps we consider are assumed to be at least of class $C^{2}$.

The study of the limit set of an end and the comprehension of the asymptotic behavior of a leaf following this end, is a difficult problem in its full generality. Many authors have studied the limit set of ends under certain conditions (see for example the introduction in [C-C1]). In particular J. Cantwell and L. Conlon have proven a theorem similar to Theorem 4 below for totally proper ends in a codimension one foliation [C-C1] as well as for isolated planar ends of proper leaves with non-exponential growth (see [C-C2, Cor. 3.4]). More precisely, they proved that totally proper leaves spiral on leaves at lower level (see in [C-Cl, section 6]). Nishimori [Ni] has studied the asymptotic behavior of isolated ends whose limit set is a compact leaf. Hector $[\mathrm{H}]$ has classified foliations for which all leaves are cylinders, furnishing us with models where cylindrical ends appear. In the present work, for the first time, we get results without the hypothesis that $L$ is proper and without any assumptions on the growth type of the leaves. Instead of these assumptions we use the compressibility of the

(C) 1997 Australian Mathematical Society 0263-6115/97\$A2.00+0.00 
cylindrical end and the hypothesis that the end is of trivial linking type (see definitions 1 and 3 below). Roughly speaking, the idea of the proof is to consider and study the foliation $\mathscr{F}_{D}$ induced by $\mathscr{F}$ on a disc $D$ associated to a compressible cylindrical end $e$.

Finally, it is not known if there are cylindrical compressible ends of non-trivial linking type. Contrarily, it seems plausible that the following must have an affirmative answer:

QUESTION. Is each cylindrical compressible end in $M$ of trivial linking type?

We will need the concept of the end of an open leaf of $\mathscr{F}$. For details concerning this concept see [C-C1, C-C2]. Let $L$ be an open leaf of $\mathscr{F}$. An end $e$ of $L$ is defined by a decreasing sequence $\cdots \supset K_{n} \supset K_{n+1} \supset \cdots$ such that:

(a) Each $K_{n}$ is a closed 2-dimensional submanifold of $L$ with boundary.

(b) The boundary $\partial K_{n}$ is diffeomorphic to the unit sphere $\mathbb{S}^{1}$.

(c) $\cap_{n} K_{n}=\emptyset$.

Such a sequence $\left(K_{n}\right)_{n=1.2 \ldots}$ defining an end $e$ is called a fundamental neighborhood system of $e$ and we write $e=\left(K_{n}\right)_{n=1.2 \ldots . . .}$.

The $e$-limit set of $L$ is defined by e-lim $(L)=\cap_{i} \overline{K_{i}}$; as usual, $\overline{K_{i}}$ denotes the closure of $K_{i}$ in $M$. It is elementary that $e-\lim (L)$ is compact and $\mathscr{F}$-satured.

An end $e=\left(K_{n}\right)_{n=1,2 \ldots}$ is called cylindrical if $K_{1}$ (and hence each $K_{i}$ ) is diffeomorphic to $\mathbb{S}^{1} \times \mathbb{R}$.

The ends of a leaf $L$ often become apparent in terms of simpler leaves on which they wind around. These results are a kind of Poincaré-Bendixson theory for foliations of codimension one. In the following we prove a theorem of this type for cylindrical compressible ends of trivial linking type. We need the following definitions.

DEFINITION 1. Let $e=\left(K_{n}\right)_{n=1,2 \ldots}$ be a cylindrical end of a leaf $L$. The end $e$ will be called compressible if there exists a 2-dimensional disc $D$ embedded in $M$ such that: $\partial D \subset K_{1}, \partial D$ is a non-contractible curve in $L$ and $D$ is transverse to $L$ along $\partial D$. Such a disc $D$, will be called a disc associated to $e$.

A simple ciosed curve of $K_{1}$ which is non-contractible in $K_{1}$ will be called a generator of $K_{1}$. Obviously any two generators of $K_{1}$ are freely homotopic in $K_{1}$.

DEFINITION 2. We will say that two disjoint generators $\gamma_{1}, \gamma_{2}$ of $K_{1}$ form a trivial link in $M$ if for any two embedded discs $D_{1}, D_{2}$ in $M$ with $\partial D_{1}=\gamma_{1}, \partial D_{2}=\gamma_{2}$ there is an isotopie $h_{t}: D_{1} \rightarrow M, 0 \leq t \leq 1$ such that:

(1) $h_{0}\left(D_{1}\right)=D_{1}, h_{1}\left(D_{1}\right)=D_{1}^{\prime}$ and $D_{1}^{\prime} \cap D_{2}=\emptyset$;

(2) $h_{t}\left(\gamma_{1}\right)=\gamma_{1}$ for each $t \in[0,1]$.

DEFINITION 3. We will say that a compressible end $e=\left(K_{n}\right)_{n=1,2 \ldots}$ is of trivial linking type if any two disjoint generators of $K_{\mathrm{I}}$ form a trivial link in $M$. 
REMARK 1. It is not difficult to prove that if two generators of $K_{1}$ form a trivial link then any two generators of $K_{1}$ form a trivial link in $M$

We may use a weaker (and more standard) definition for the trivial link between two generators of $K_{1}$ (see for example [Ro]). If we do so, the hypothesis $\pi_{2}(M)=0$ must be added in the theorem below. In any case we will not discuss this alternative as it is beyond the scope of this work.

The aim of this paper is to prove:

THEOREM 4. Let $\mathscr{F}$ be a 2-dimensional orientable foliation on a compact orientable 3-manifold $M$. Assume that each embedded 2-sphere in $M$ separates $M$ (that is, the complement of the embedded sphere in $M$, consists of exactly two components). Let $e=\left(K_{n}\right)_{n=1,2 \ldots}$ be a cylindrical compressible end of trivial linking type of a leaf $L \in \mathscr{F}$, where $L \neq \mathbb{R}^{2}$. Then there exists a smooth embedding $f: \mathbb{S}^{1} \times[0, \varepsilon) \rightarrow M$ and a decreasing sequence $\left(t_{n}\right), 0<t_{n}<\varepsilon$, converging to zero such that

(i) For all $p \in \mathbb{S}^{1}, f(\{p\} \times[0, \varepsilon))$ is an arc transverse to $\mathscr{F}$.

(ii) For all $n \in \mathbb{N}, f\left(\mathbb{S}^{1} \times\left\{t_{n}\right\}\right)=c_{n} \subset K_{1}$ and $c_{n}$ is non-contractible in $L$.

(iii) The curve $f\left(\mathbb{S}^{1} \times\{0\}\right)=c_{0}$ belongs to a toral leaf $L_{0}$ and $c_{0}$ is noncontractible in $L_{0}$. Moreover, $e$ - $\lim (L)=L_{0}$.

\section{Proof of the theorem}

Choose a point $y_{0} \in \mathrm{e}-\lim (L)$ such that $y_{0} \notin L$. Such a point exists since, by compactness of e-lim(L), one has $L \neq \mathrm{e}-\lim (L)$. Therefore there exists a sequence of points $\left(y_{n}\right)_{n=1,2, \ldots}$ such that:

(i) $\mathrm{y}_{n} \in K_{n}, n=1,2, \ldots$

(ii) $y_{n}$ converges to $y_{0}$ along an arc $S$ transverse to $\mathscr{F}$.

For each $n=1,2, \ldots$ we consider a simple closed curve $\beta_{n}$ in $K_{n}$ which contains $y_{n}$ and is not contractible in $L$. Moreover we choose the curves $\beta_{n}$ to be pairwise disjoint.

Since $h: \mathbb{D}^{2} \rightarrow M$ is a $C^{r}$-map we may assume that $h$ is in general position with respect to $\mathscr{F}$. That is to say, $h$ is transverse to $\mathscr{F}$ in a neighborhood of $\partial \mathbb{D}^{2}$ and the foliation $h^{*} \mathscr{F}$, induced on $\mathbb{D}^{2}$ from $\mathscr{F}$ by the map $h$, contains singularities of saddle or central type. Fix a transverse orientation on $\mathscr{F}$ and let $\eta$ (respectively $\eta_{h^{*}}$ ) be the unit vector field normal to $\mathscr{F}$ (respectively $h^{*} \mathscr{F}$ ). Let $\left[y_{1}, y_{0}\right]=J \subset S$ be the segment between the points $y_{1}$ and $y_{0}$. Then we have

LEMMA 5. There exists an embedding $h: \mathbb{D}^{2} \rightarrow M$ in general position with respect to $\mathscr{F}$ such that $h\left(\partial \mathbb{D}^{2}\right)=\beta_{1}$ and $J \subset h\left(\mathbb{D}^{2}\right)$. Moreover, if $l_{k}^{\prime}$ is the leaf of $h^{*} \mathscr{F}$ passing through $h^{-1}\left(y_{k}\right)$, then $h\left(l_{k}^{\prime}\right)$ cannot be a closed curve contractible in $L$ for any $k \in \mathbb{N}$. 
PROOF. Since the end $e$ is compressible, there exists an embedding $h_{0}: \mathbb{D}^{2} \rightarrow M$ in general position with respect to $\mathscr{F}$ such that $h_{0}\left(\partial \mathbb{D}^{2}\right)=\beta_{1}$. If $J \subset h_{0}\left(\mathbb{D}^{2}\right)$, set $h=h_{0}$. If not, then we may assume that $J$ intersects $h_{0}\left(\mathbb{D}^{2}\right)$ transversely in a finite number of points. Therefore, by a deformation of $h_{0}\left(\mathbb{D}^{2}\right)$ in $M$, we may assume that $h_{0}: \mathbb{D}^{2} \rightarrow M$ is an embedding such that:

(i) $h_{0}\left(\partial \mathbb{D}^{2}\right)=\beta_{1}^{\prime}$ where $\beta_{1}^{\prime} \subset K_{1}$;

(ii) $\beta_{1}^{\prime} \cap \beta_{1}=\emptyset$ and $\beta_{1}^{\prime}$ is freely homotopic to $\beta_{1}$ in $K_{1}$;

(iii) $J \cap h_{0}\left(\mathbb{D}^{2}\right)=\emptyset$.

In the following we consider an embedding $h_{1}: \mathbb{S}^{1} \times[0,1] \rightarrow M$ which satisfies:

(i) $h_{1}\left(\mathbb{S}^{1} \times\{0\}\right)=\beta_{1}$ and $J \subset h_{1}\left(\mathbb{S}^{1} \times[0,1 / 2]\right)$;

(ii) $h_{1}$ is transverse to $\mathscr{F}$ in a neighborhood of $\beta_{1} \cup J$;

(iii) $h_{1}\left(\mathbb{S}^{1} \times[0,1]\right) \cap h_{0}\left(\mathbb{D}^{2}\right)=\emptyset$.

The curve $h_{1}\left(\mathbb{S}^{1} \times\{1\}\right)=\beta_{2}^{\prime}$ is homotopic to $\beta_{1}^{\prime}$ and, obviously there exists an embedding $h_{2}: \mathbb{S}^{1} \times[0,1] \rightarrow M$ such that:

(i) $h_{2}\left(\mathbb{S}^{1} \times\{0\}\right)=\beta_{2}^{\prime}, h_{2}\left(\mathbb{S}^{1} \times\{1\}\right)=\beta_{1}^{\prime}$;

(ii) $h_{2}\left(\mathbb{S}^{1} \times(0,1)\right) \cap h_{0}\left(\mathbb{D}^{2}\right)=\emptyset$;

(iii) $h_{2}\left(\mathbb{S}^{1} \times(0,1)\right) \cap h_{1}\left(\mathbb{S}^{1} \times[0,1]\right)=\emptyset$.

By gluing the maps $h_{2}, h_{1}$ along $\beta_{2}^{\prime}$ and $h_{1}, h_{0}$ along $\beta_{1}^{\prime}$ we get a continuous injection $f: \mathbb{D}^{2} \rightarrow M$ with $f\left(\partial \mathbb{D}^{2}\right)=\beta_{1}$ and such that:

(i) $f$ is differentiable in a neighborhood $V$ of $\beta_{1}$ with $J \subset V$;

(ii) $f$ is transverse to $\mathscr{F}$ in a neighborhood $U$ of $\beta_{1} \cup J$ with $U \subset V$.

By rounding the corners, we can replace $f$ by a $\left(C^{r}-\right)$ embedding $f_{0}: \mathbb{D}^{2} \rightarrow M$ which coincides with $f$ in a neighborhood of $\partial \mathbb{D}^{2}$ and such that:

(i) $J$ is contained in $f_{0}\left(\mathbb{D}^{2}\right)$;

(ii) $f_{0}$ is transverse to $\mathscr{F}$ in a neighborhood of $\beta_{1} \cup J$.

Now it is well known (see [G, Ch. IV, Lemma 1.6]) that for every $\varepsilon>0$ we can find a $C^{r}$-map $h: \mathbb{D}^{2} \rightarrow M$, in general position with respect to $\mathscr{F}, \varepsilon$-near to $f_{0}$, which coincides with $f_{0}$ in a neighborhood of $\partial \mathbb{D}^{2} \cup f_{0}^{-1}(J)$. This map $h$ is an embedding since $\varepsilon$ can be chosen arbitrarily small and it satisfies the conditions of Lemma 5 .

In the following, the theorem of Novikov concerning the existence of Reeb components [N] easily implies that if some curve $l_{k}^{\prime}$ is closed then its image $h\left(l_{k}^{\prime}\right)$ cannot be homotopic to zero in $L$. In fact: suppose that $h\left(l_{k}^{\prime}\right)$ is homotopic to zero in $L$. Then we can find a differentiable map $G: \mathbb{S}^{1} \times[0,1] \rightarrow M$ such that:

(a) $G\left(\mathbb{S}^{1} \times[0,1]\right) \subset h\left(\mathbb{D}^{2}\right)$ and for every $t \in[0,1]$, the curve $g_{t}: \mathbb{S}^{1} \rightarrow M$, defined by $g_{t}(x)=G(t, x)$, is contained in a leaf $L_{t}$ of $\mathscr{F}$. Moreover $g_{0}\left(\mathbb{S}^{1}\right)=h\left(l_{k}^{\prime}\right)$.

(b) For every $x \in \mathbb{S}^{1}$, the curve $t \rightarrow G(t, x)$ is transverse to $\mathscr{F}$ and the orientation of this curve is opposite to the transverse orientation of $\mathscr{F}$ induced by the normal field $\eta$. 
(c) For every $t \in[0,1)$, the curve $g_{t}$ is homotopic to a constant in $L_{t}$ and $g_{1}$ is not homotopic to a constant in $L_{1}$.

From Novikov's theorem mentioned above, the leaf $L_{1}$ must be a toral leaf which bounds a solid torus $T$ in $M$ and all leaves $L_{t}$ are diffeomorphic to planes contained in the interior of $T$. Therefore, the leaf $L$ must be a plane contained in the interior of $T$. Obviously, this is impossible since there are points of $L$ outside of $T$.

Next, we will prove the following:

PROPOSITION 6. There exists an embedding $g: \mathbb{D}^{2} \rightarrow M$ in general position with respect to $\mathscr{F}$ with $g\left(\partial \mathbb{D}^{2}\right)=\beta_{1}$ and such that:

(1) There is an arc $J^{\prime}$ transverse to $g^{*} \mathscr{F}$ with $g\left(J^{\prime}\right)=J$, and a sequence $y_{n}^{\prime}$ in $J^{\prime}$ with $g\left(y_{n}^{\prime}\right)=y_{n}$, such that all the leaves of $g^{*} \mathscr{F}$ passing through $y_{n}^{\prime}, n=2,3, \ldots$, are simple closed curves. We denote these curves by $a_{n}^{\prime}$. The points $y_{n}^{\prime}$ converge to a point $y_{0}^{\prime} \in J^{\prime}$.

(2) If $a_{n}=g\left(a_{n}^{\prime}\right)$ then $a_{n} \subset L$ for $n=1,2, \ldots$ and they are non-contractible curves in $L$. For each $n$, there is a component $V_{n}$ of $L-a_{n}$ such that $K_{1} \supset \mathrm{cl} V_{1} \supset$ $\operatorname{cl} V_{2} \supset \cdots \supset \mathrm{cl} V_{n} \supset \cdots$ and each $\mathrm{cl} V_{n}$ is diffeomorphic to $\mathbb{S}^{1} \times[0, \infty)$; here by cl $V_{n}$ we denote the closure of $V_{n}$ in $L$.

REMARK 2. We may assume that such an embedding $g: \mathbb{D}^{2} \rightarrow M$ intersects $L$ transversely. In fact, $g^{*} \mathscr{F}$ has a finite number of singularities. So if the leaves $l$ induced by $L$ on $g\left(\mathbb{D}^{2}\right)$ contain saddle points we displace them to leaves next to $l$ by a small perturbation of $g\left(\mathbb{D}^{2}\right)$ in $M$.

Assuming Proposition 6 is not true we obtain a contradiction with the aid of Lemmas 7, 8 and 9 which follow. In fact, suppose that Proposition 6 is not true. Then we have

LEMMA 7. If Proposition 6 is not true, there is an embedding $g: \mathbb{D}^{2} \rightarrow M$ in general position with respect to $F$ such that:

(1) There is an arc $J^{\prime}$ transverse to $g^{*} \mathscr{F}$ with $g\left(J^{\prime}\right)=J$ and a sequence of points $y_{n}^{\prime}$ in $J^{\prime}$ with $g\left(y_{n}^{\prime}\right)=y_{n}$ converging to $y_{0}^{\prime}$ following the positive direction of $J^{\prime}$.

(2) There is an $i \in\{2,3, \ldots\}$ such that the leaves $a_{m}^{\prime}$ of $g^{*} \mathscr{F}$ passing through $y_{m}^{\prime}$ for $m<i$, are simple closed curves and their images $a_{m}=g\left(a_{m}^{\prime}\right)$ are simple and non-contractible in L. Otherwise the leaf $l_{i}^{\prime}$ passing through $y_{i}^{\prime}$ is homeomorphic to $\mathbb{R}$.

(3) There is a component $V_{n}$ of $L-a_{n}, n=1,2, \ldots, i-1$ such that $K_{1} \supset$ $\operatorname{cl} V_{1} \supset \mathrm{cl} V_{2} \supset \cdots \supset \mathrm{cl} V_{i-1}$, and each $\mathrm{cl} V_{n}, n=1,2, \ldots, i-1$ is diffeomorphic to $\mathbb{S}^{1} \times[0, \infty)$.

(4) The natural number $i$ posited in (2) above is the smallest natural for which there exists an embedding $g: \mathbb{D}^{2} \rightarrow M$ in general position with respect to $\mathscr{F}$ which satisfies the conditions (1)-(3) of the lemma. 
PROOF. If the conditions (1)-(3) of Lemma 7 were satisfied for each $i \in\{2,3, \ldots\}$ then Proposition 6 would be valid. On the other hand Lemma 5 guarantees that for $i=2$ there exists a map $g: \mathbb{D}^{2} \rightarrow M$ in general position with respect to $\mathscr{F}$ which satisfies conditions (1)-(3). Therefore if Proposition 6 is not true there exists an $i \geq 2$ for which conditions (1)-(4) are satisfied.

LEMMA 8. Let $l_{i}=g\left(l_{i}^{\prime}\right)$ and let $r(t), t \in(-\infty,+\infty)$, be a parametrization of $l_{i}$. Set $l_{i}^{+}=r([0,+\infty)), l_{i}^{-}=r((-\infty, 0])$. Then $l_{i}^{+}, l_{i}^{-}$intersect all curves $\beta_{n}$ of $L$ for $n>i$.

PROOF. The curve $l_{i}$ does not intersect $\beta_{1}$ since $\mathscr{F}$ is tangent to $g\left(\partial \mathbb{D}^{2}\right)=\beta_{1}$. Suppose now that $l_{i}^{+}$does not intersect all the curves $\beta_{n}$ for $n>i$. Then there is an annulus $C$ in $L\left(C \approx \mathbb{S}^{1} \times[0,1]\right)$ such that

$$
l_{i}^{+} \cap C \neq \emptyset \quad \text { and } \quad l_{i}^{+} \cap \partial C=\emptyset .
$$

In fact, there exists a natural number $k>i$ such that $l_{i}^{+} \cap \beta_{k}=\emptyset$. Note also that the point $y_{i}$ of $l_{i}^{+}$belongs to $K_{1} \cap \beta_{i}$. Thus, if we consider the annulus $C$ in $K_{1}$ with $\partial C=\beta_{1} \cup \beta_{k}$, we have that $l_{i}^{+} \subset$ int $C$.

On the other hand, as we mentioned in Remark $2, g$ intersects $L$ transversely. Therefore $g\left(\mathbb{D}^{2}\right) \cap C$ is a compact submanifold of $C$. This implies that $l_{i}^{+}$must intersect the boundary $\partial C$ of $C$, which gives a contradiction; similarly if we consider $l_{i}^{-}$.

LEMMA 9. There is an arc $\left[z^{\prime}, w^{\prime}\right]$ transverse to $g^{*} \mathscr{F}$ such that the points $z=g\left(z^{\prime}\right)$, $w=g\left(w^{\prime}\right)$ belong to $\beta_{j}$ for some $j>i$.

PROOF. Lemma 8 asserts that $l_{i}=g\left(l_{i}^{\prime}\right)$ intersects all curves $\beta_{n}$ for $n>i$. Fix $j>i$. Let $w \in l_{i}^{+} \cap \beta_{j}, z \in l_{i}^{-} \cap \beta_{j}$ and let $z^{\prime}, w^{\prime} \in l_{i}^{\prime}$ be such that $g\left(z^{\prime}\right)=z$, $g\left(w^{\prime}\right)=w$.

Since $l_{i}^{\prime}$ is a non-compact and non-singular leaf of $g^{*} \mathscr{F}$, we can choose a neighborhood $V$ in $\mathbb{D}^{2}$ diffeomorphic to $[0,1] \times[0,1]$ and points $x^{\prime}, y^{\prime} \in l_{i}^{\prime}$ (see Figure 1) such that:

(i) $\left(g^{*} \mathscr{F}\right)_{V}$ is the product foliation $[0,1] \times\{t\}, t \in[0,1]$;

(ii) if $\left(l_{i}^{\prime}\right)^{+}$is the subset of $l_{i}^{\prime}$ with $g\left(\left(l_{i}^{\prime}\right)^{+}\right)=l_{i}^{+}$, then $\left(l_{i}^{\prime}\right)^{+} \cap V$ has an infinite number of connected components;

(iii) $x^{\prime}, y^{\prime}$ lie in different components of $\left(l_{i}^{\prime}\right)^{+} \cap V$;

(iv) if $r^{\prime}(t), t \in(-\infty,+\infty)$ is a parametrization of $l_{i}^{\prime}$ and $r^{\prime}\left(t_{1}\right)=z^{\prime}, r^{\prime}\left(t_{2}\right)=w^{\prime}$, $r^{\prime}\left(t_{3}\right)=x^{\prime}, r^{\prime}\left(t_{4}\right)=y^{\prime}$, then $t_{1}<t_{2}<t_{3}<t_{4}$.

Let $l_{i}^{\prime}\left[z^{\prime}, x^{\prime}\right]$ (respectively $l_{i}^{\prime}\left[w^{\prime}, y^{\prime}\right]$ ) denotes the subarc of $l_{i}^{\prime}$ with endpoints $z^{\prime}, x^{\prime}$ (respectively $w^{\prime}, y^{\prime}$ ). It is well known that there are tubular neighborhoods $Z$ of 
$l_{i}^{\prime}\left[z^{\prime}, x^{\prime}\right]$ and $W$ of $l_{i}^{\prime}\left[w^{\prime}, y^{\prime}\right]$ in $\mathbb{D}^{2}$ such that $\left(g^{*} \mathscr{F}\right)_{Z}$ (respectively $\left.\left(g^{*} \mathscr{F}\right)_{W}\right)$ is the product foliation.

Consider an arc $\left[x^{\prime}, y^{\prime}\right]$ transverse to $\left(g^{*} \mathscr{F}\right)_{V}$. Now we can find disjoint subarcs $\left[x^{\prime}, x^{\prime \prime}\right],\left[y^{\prime \prime}, y^{\prime}\right]$ of $\left[x^{\prime}, y^{\prime}\right]$, as well as arcs $\left[z^{\prime}, x^{\prime \prime}\right]$ and $\left[w^{\prime}, y^{\prime \prime}\right]$ transverse to $\left(g^{*} \mathscr{F}\right)_{z}$ and $\left(g^{*} \mathscr{F}\right)_{W}$ respectively such that if $\left[x^{\prime \prime}, y^{\prime \prime}\right] \subset\left[x^{\prime}, y^{\prime}\right]$, then the $\operatorname{arcs}\left[x^{\prime \prime}, y^{\prime \prime}\right],\left[z^{\prime}, x^{\prime \prime}\right]$ and $\left[w^{\prime}, y^{\prime \prime}\right]$ are pairwise disjoint (see for example Figure 1). The union $\left[z^{\prime}, x^{\prime \prime}\right] \cup$ $\left[x^{\prime \prime}, y^{\prime \prime}\right] \cup\left[y^{\prime \prime}, w^{\prime}\right]$ is an arc in $\mathbb{D}^{2}$ transverse to $g^{*} \mathscr{F}$.

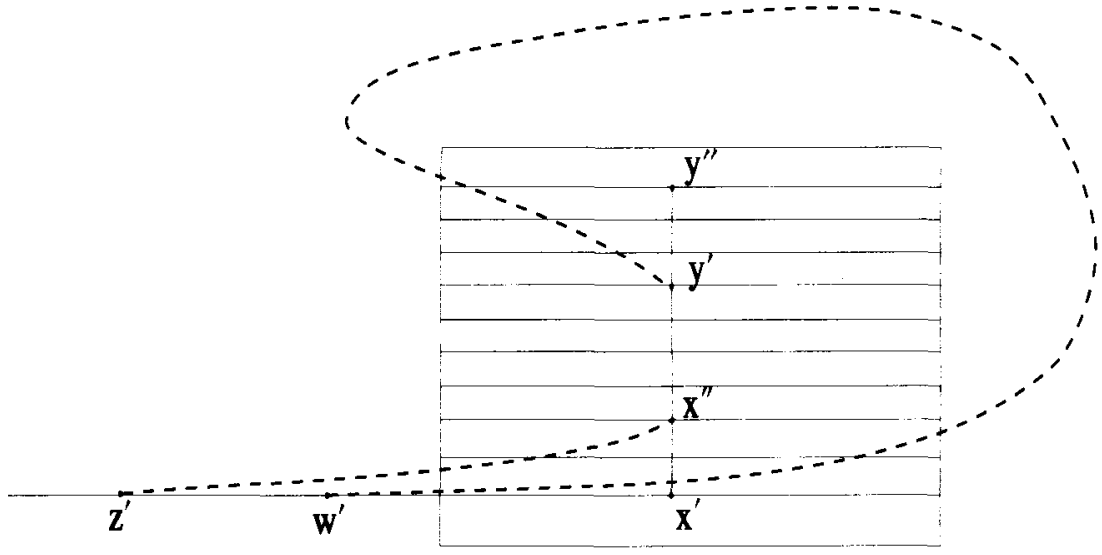

FIGURE 1

Now there exists a disc $D$ embedded in $M$ with $\partial D=\beta_{j}$ and $D \cap g\left(\mathbb{D}^{2}\right)=\emptyset$. This follows from the fact that $g\left(\partial \mathbb{D}^{2}\right)=\beta_{1}$ and $\beta_{1}, \beta_{j}$ form a trivial link. In what follows we can choose disjoint generators $\beta_{j}^{\prime}, \beta_{j}^{\prime \prime}$ of $K_{1}$ sufficiently near to $\beta_{j}$ such that if $L_{j}$ is the annulus in $K_{1}$ with $\partial L_{j}=\beta_{j}^{\prime} \cup \beta_{j}^{\prime \prime}$ then $\beta_{j} \subset$ int $L_{j}$ and $L_{j} \cap D=\beta_{j}$. We have the following:

ClaIM. We can choose discs $D_{j}^{\prime}, D_{j}^{\prime \prime}$ embedded in $M$ such that:

(i) $\partial D_{j}^{\prime}=\beta_{j}^{\prime}, \partial D_{j}^{\prime \prime}=\beta_{j}^{\prime \prime}$;

(ii) $D_{j}^{\prime} \cap g\left(\mathbb{D}^{2}\right)=\emptyset, D_{j}^{\prime \prime} \cap g\left(\mathbb{D}^{2}\right)=\emptyset$;

(iii) $S=L_{j} \cup D_{j}^{\prime} \cup D_{j}^{\prime \prime}$ is a topological 2-sphere which bounds a 3-ball $B$ in $M$.

PROOF OF CLAIM. We consider a tubular neighbourhood $V$ of $L_{j}$ in $M$ diffeomorphic to $L_{j} \times(-1,+1)$, where $L_{j} \approx L_{j} \times\{0\}$ under this diffeomorphism. We consider also a neighborhood $W$ of $D$ diffeomorphic to $D \times(-1,+1)$ such that $\partial D \times(-1,+1) \subset L_{j}$ and $W \cap g\left(\mathbb{D}^{2}\right)=\emptyset$. Therefore, we can construct the discs 
$D_{j}^{\prime}, D_{j}^{\prime \prime}$ of the claim within the union $V \cup W$. In Figure $2\left(\right.$ a) the discs $D_{j}^{\prime}, D_{j}^{\prime \prime}$ are designated by thick arcs.

From the previous claim we have that $S$ separates $M$ into two connected components. Now we examine the relative position of $g\left(\left[z^{\prime}, w^{\prime}\right]\right)$ with respect to $S$. All vectors of $\eta$ on $L_{j}$ are pointing either inwards or outward of the component $B$ and $g\left(\left[z^{\prime}, w^{\prime}\right]\right)$ is transverse to $\mathscr{F}$. Therefore there exists an $\varepsilon>0$ such that $g\left(\left[z^{\prime}, z^{\prime}+\varepsilon\right]\right)$ is contained in $B$ and $g\left(\left(w^{\prime}, w^{\prime}-\varepsilon\right]\right)$ is contained in the complement of $B$. Since $g\left(\left[z^{\prime}, w^{\prime}\right]\right)$ is transverse to $\mathscr{F}$ we may assume that $g\left(\left[z^{\prime}, w^{\prime}\right]\right) \cap D_{j}^{\prime \prime} \neq \emptyset$ or $g\left(\left[z^{\prime}, w^{\prime}\right]\right) \cap D_{j}^{\prime} \neq \emptyset$. However, by construction of the arc $g\left(\left[z^{\prime}, w^{\prime}\right]\right)$ and of the discs $D_{j}^{\prime}, D_{j}^{\prime \prime}, g\left(\left[z^{\prime}, w^{\prime}\right]\right)$ intersects neither $D_{j}^{\prime}$ nor $D_{j}^{\prime \prime}$; this gives the promised contradiction.

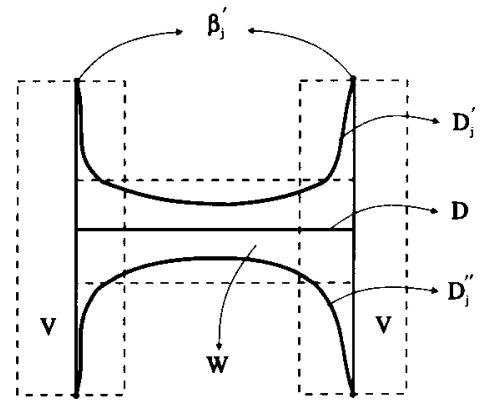

(a)

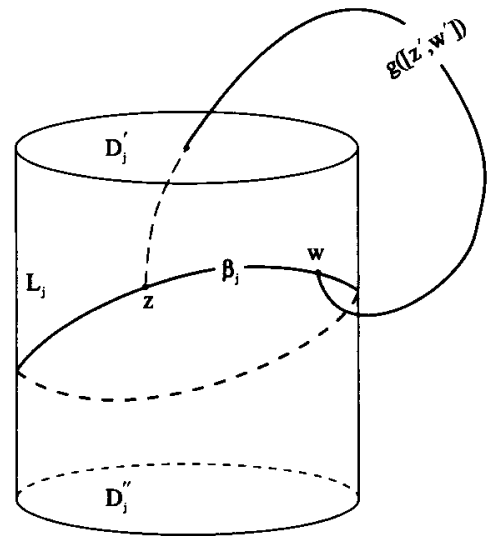

(b)

FIGURE 2

Therefore, Proposition 6 is valid. So we deduce immediately the following:

LEMMA 10. There exists an embedding $f: \mathbb{S}^{1} \times[0,1) \rightarrow M$ and a sequence $t_{n} \in[0,1)$ decreasing to 0 such that:

(1) If $f_{t}=f(\cdot, t), t \in[0,1)$ then $f_{t_{n}}\left(\mathbb{S}^{1}\right)=a_{n} \subset L$ and $f_{0}\left(\mathbb{S}^{1}\right)=a_{0} \subset L_{0}$.

(2) For each $p \in \mathbb{S}^{1}$ the curve $f_{t}(p), t \in[0,1)$, is transverse to $\mathscr{F}$.

PROOF. From proposition 6 we have that the sequence of closed curves $a_{n}$ of $L$ converges uniformly to a closed curve $a_{0}$ of a leaf $L_{0}$. Obviously $a_{0}$ is non-contractible in $L_{0}$ since all the curves $a_{n}$ are non-contractible in $L$. Therefore we can obtain easily the family of embeddings $f_{t_{n}}\left(\mathbb{S}^{1}\right)$ which satisfies the properties 1,2 of the lemma. Note that here we are using the hypothesis that $\mathscr{F}$ is transversely orientable. 
Now we will prove that $L_{0}$ is a torus in $M$. In order to prove this we work similarly to the appendix of [R-R], where it is proved that the existence of a vanishing cycle implies the existence of a Reeb component. For this proof we omit some details, and we refer to $[R-R]$ for them.

\section{LEMMA 11. The leaf $L_{0}$ is homeomorphic to a torus.}

PROOF. In order to prove that $L_{0}$ is compact it suffices to show that there is no closed transversal curve intersecting $L_{0}$. Suppose $\gamma$ is a closed transversal curve which intersects $L_{0}$ at a point $p$. We may assume that $\gamma$ coincides with the segment $\left\{f_{t}(p), 0 \leq t \leq \varepsilon\right\}$ where $\varepsilon<1$ and we orient $\gamma$ in the positive sense of the foliation $\mathscr{F}$. Choose $t_{m}, t_{n}: 0<t_{m}<t_{n}<\varepsilon$. Let $L(m, n) \subset L$ be the annulus limited by $a_{m}, a_{n}$ and let $D(m, n) \subset g\left(\mathbb{D}^{2}\right)$ be the annulus limited by the same curves. Then $T(m, n)=L(m, n) \cup D(m, n)$ is a torus and we will prove that $T(m, n)$ separates $M$ in two connected components. In fact, consider a disc $D$ embedded in $M$ such that $D \cap T(m, n)=d$ is a generator of $L(m, n)$. Such a disc exists since the end $e$ is compressible and of trivial linking type. Let now $D^{\prime}$ be a disc parallel to $D$ such that:

(i) $D^{\prime} \cap L(m, n)=d^{\prime}$ and $d^{\prime}$ is a generator of $L(m, n)$ disjoint from $d$.

(ii) If $A$ is the annulus in $L(m, n)$ with $\partial A=d \cup d^{\prime}$ then $A \cup D \cup D^{\prime}$ is a 2-sphere which bounds a 3-ball in $M$.

If $A^{\prime}=L(m, n)-A$ then $A^{\prime} \cup D \cup D^{\prime}$ is a 2-sphere which separates $M$ by hypothesis. Therefore $T(m, n)$ separates $M$.

Denote by $S(m, n)$ the component of $M-T(m, n)$ such that all the positive normals point into $S(m, n)$ along $L(m, n)$. This implies that $\gamma(t) \in S(m, n)$ for $t>t_{n}$ and therefore $L_{0}$ is compact. Now, since the curve $a_{0}$ is non-contractible in $L_{0}$ it follows that $L_{0}$ is a leaf of genus greater than 0 .

Finally as in [R-R, Lemma 5] we define a diffeomorphism $\Phi(m, n): L_{0}-f_{0}\left(S^{l}\right) \rightarrow$ $L(m, n)$ which proves that $L_{0}$ is a torus.

\section{Acknowledgment}

The author would like to thank Professor G. Hector for very helpful discussions and the referee for his important suggestions towards the clarity of this paper.

\section{References}

[C-C1] J. Cantwell and L. Conlon 'Poincaré-Bendixson theory for leaves of codimension one', Trans. Amer. Math. Soc. (1981), 181-209.

[C-C2] - 'Leaves with isolated ends in foliated 3-manifolds', Topology 16 (1972), 311-322. 
[G] C. Godbillon, Théorie géométrique des feuilletages (Birkhäuser, Boston, 1992)

[H] G. Hector, 'Feuilletages en cylindres', in: Lecture Notes in Math. 597 (Springer, Berlin, 1977) pp. 252-270.

[N] S. P. Novikov, 'Topology of foliations', Trans. Moscow Math. Soc. (1965), 268-304.

[Ni] T. Nishimori, 'Isolated ends of open leaves of codimension one foliations', Quart. J. Math. Oxford 26 (1975), 159-167.

[Ro] D. Rolfsen, Knots and links (Publish or Perish, Inc., Houston, 1976).

[R-R] H. Rosenberg and R. Roussarie, 'Reeb foliations', Ann. of Math. 91 (1970), 1-24.

Agricultural University of Athens

Department of Mathematics

75 Iera Odos, 11855

Athens

Greece

e-mail: gmat2xax@ auadec.aua.ariadne-t.gr 